\title{
Beatmung und Sekretmanagement bei amyotropher Lateralsklerose ${ }^{1}$
}

\author{
Ventilatory Support and Management of Secretions in \\ Amyotrophic Lateral Sclerosis
}

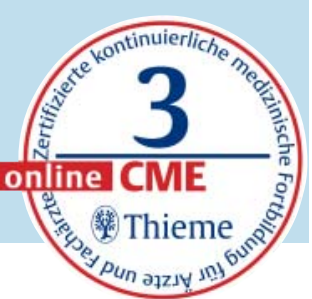

Klinik für Schlafmedizin und Neuromuskuläre Erkrankungen, Universitätsklinikum Münster

VNR

2760512016149751707

\section{Bibliografie}

DOI http://dx.doi.org/ $10.1055 / \mathrm{s}-0042-117284$

Fortschr Neurol Psychiatr 2016; 84: 640-650 @ Georg Thieme Verlag KG Stuttgart · New York . ISSN 0720-4299

\section{Korrespondenzadresse}

Dr. med. Matthias Boentert

Universitätsklinikum Münster, Department für Neurologie, Klinik für Schlafmedizin und Neuromuskuläre Erkrankungen Albert-Schweitzer-Campus 1 48149 Münster matthias.boentert@ ukmuenster.de

\section{Zusammenfassung}

Die Amyotrophe Lateralsklerose (ALS) ist eine neurodegenerative Systemerkrankung mit variablem, aber meist raschem Krankheitsverlauf und einer immer noch infausten Prognose, die wesentlich von der bei nahezu allen Patienten eintretenden Parese der Atemmuskulatur und ihren Folgen bestimmt wird. Angesichts begrenzter kausaler Therapiemöglichkeiten kommt einer umfassenden und qualifizierten symptomorientierten Behandlung eine zentrale Rolle zu. Symptome einer Zwerchfellschwäche, einer schlafbezogenen Hypoventilation und im Verlauf einer chronischen hyperkapnischen respiratorischen Insuffizienz haben erheblichen Einfluss auf die gesundheitsbezogene Lebensqualität und die Teilhabefähigkeit der betroffenen Patienten. Im vorliegenden Übersichtsartikel wird systematisch auf die klinische Präsentation der Atemmuskelschwäche, Methoden zur (Früh-)Diagnose sowie praktische Gesichtspunkte und bewiesene Effekte der Beatmungstherapie und Hustenassistenz eingegangen. Der diagnostische Einsatz technischer und elektrophysiologischer Methoden hat die Feststellung der Atempumpenschwäche und der schlafbezogenen Hypoventilation zum Ziel. Hierbei finden spiromanometrische Methoden zur direkten oder indirekten Messung der Atemmuskelkraft, die kardiorespiratorische Polygraphie und Polysomnografie, die transkutane Kapnometrie und die Blutgasanalyse Anwendung. Therapeutisch haben die nicht-invasive Beatmung, bei entsprechendem Patientenwunsch die invasive Beatmung über eine Trachealkanüle und das Sekretmanagement einen im Krankheitsverlauf zunehmenden Stellenwert.

\section{Abstract \\ $\nabla$}

The term amyotrophic lateral sclerosis (ALS) comprises a group of motor neuron diseases which are characterized by rapid disease progression and poor prognosis which is mostly due to severe respiratory muscle weakness and its sequelae. Since causative treatment options are limited it is crucial to offer comprehensive symptomatic therapies to affect patients. Symptoms of respiratory muscle weakness, sleep-disordered breathing and, subsequently, chronic hypercapnic respiratory failure are known to severely affect health-related quality of life and social functioning of patients with ALS. This review article delineates the clinical presentation of respiratory muscle weakness, diagnostic procedures to assess diaphragmatic function, and practical aspects of both mechanical ventilation and cough assistance, respectively. Various technical and electrophysiological methods allow for detection of diaphragmatic weakness and nocturnal hypoventilation. These include spiro-manometric tests of respiratory muscle strength, cardiorespiratory polygraphy and polysomnography, transcutaneous capnography, and blood gas analysis. Once the diagnosis of respiratory muscle weakness is established, non-invasive ventilation, tracheostomyinvasive ventilation (if the patient agrees to it), and management of secretions all become increasingly important in the course of the disease.

\footnotetext{
${ }^{1}$ Erstveröffentlichung in Klin Neurophys 2015; 46: 163 172.
} 


\section{Lernziele}

$\nabla$

Kenntnis der

> klinischen Symptome schlafbezogener Atmungsstörungen und der muskulären Atemschwäche

- spirometrischen, manometrischen und schlafmedizinischen Untersuchungsverfahren zur Detektion einer muskulären Ateminsuffizienz und einer schlafbezogenen Atmungsstörung

- Indikationskriterien für die Einleitung einer nicht invasiven Heimbeatmung

- Effekte von Beatmungstherapie auf Lebensqualität und Lebenserwartung von Patienten mit ALS

> Indikationskriterien und Durchführung manueller und technischer Hilfen zur Unterstützung des Hustens bei neuromuskulärer Atemschwäche

\section{Einführung \\ $\nabla$}

Die amyotrophe Lateralsklerose (ALS) ist eine neurodegenerative Multisystemerkrankung, die mit einem fortschreitenden Funktionsverlust motorischer Neurone und variabel ausgeprägten neurokognitiven Defiziten bis hin zur frontotemporalen Demenz (FTD) verbunden ist. Die Inzidenz der Erkrankung beträgt ca. 0,6 - 2,4/100 000, die Prävalenz wird mit 5-8/100000 angegeben [1-3]. Die ALS kann sich ab dem Jugendalter manifestieren, der Altersgipfel liegt jedoch zwischen dem 50. und 70. Lebensjahr. Männer sind ca. 1,6mal häufiger betroffen als Frauen [2, 3]. Bei 85 $90 \%$ der Betroffenen tritt die Erkrankung sporadisch auf. Familiäre Formen der ALS folgen meist einem dominanten Erbgang.

Symptome Die ALS ist eine Motoneuronerkrankung mit Beteiligung sowohl des 1. als auch des 2. Motoneurons, sodass das klinische Bild durch das gemeinsame Auftreten von spastischen Paresen und Hyperreflexie mit muskulärer Atrophie, Faszikulationen und Bulbärparalyse gekennzeichnet ist. Je nach initialer Lokalisation und Schwerpunkt der Symptome wird zwischen einer bulbären und einer spinalen Verlaufsform der ALS unterschieden; im Verlauf entwickeln sich häufig Mischformen.

Verlauf Der Untergang von Vorderhornzellen im zervikalen Myelon führt zum Axonverlust im N. phrenicus, Plexus cervicalis und den Interkostalnerven, woraus eine fortschreitende Schwäche des Zwerchfells und der Atemhilfsmuskulatur resultiert. Dies führt zu einer in- und exspiratorischen Muskelschwäche, sodass Hypoventilation und Husteninsuffizienz häufig Hand in Hand gehen. Die chronische respiratorische Insuffizienz tritt bei wenigen Patienten als Erstsymptom [4] und bei nahezu allen Patienten im Erkrankungsverlauf auf. Sie ist ein wesentlicher Grund für die schlechte Prognose und die häufigste Todesursache bei ALS [5]. Betroffene Patienten sterben in der Regel an einer Hyperkapnie oder Hypoxämie im Rahmen der alveolären Hypoventilation, die oft durch respiratorische Infekte, Aspiration und Sekretverhalt verstärkt wird. Die mittlere Lebenserwartung beträgt nach Symptombeginn 3-5 Jahre [6], nach Diagnosestellung nur 1,5 - 2 Jahre $[7,8]$.

Therapie Angesichts der Tatsache, dass über die Medikation mit dem Glutamatantagonisten Riluzol hinaus keine kausalen Behandlungsmöglichkeiten zur Verfügung stehen, kommt der symptomatischen Therapie eine zentrale Bedeutung zu. Sie hat zum einen die Verbesserung der Lebensqualität in jeder Krankheitsphase zum Ziel und nimmt zum anderen im besten Fall einen verzögernden Einfluss auf den Krankheitsverlauf. Ein umfassender und vollständiger symptomorientierter Behandlungsansatz der ALS hat dem biopsychosozialen Modell [9] entsprechend die Problemfelder Mobilität, Kommunikation, Psyche und Soziales, Schmerzen, Atmung und Ernährung im Blick. Der Stellenwert des Themas „Atmung und Beatmung“ ergibt sich aus den vitalen Konsequenzen der respiratorischen Insuffizienz.

Im Folgenden wird systematisch auf die klinische Manifestation der Atemmuskelschwäche, Methoden zur (Früh-)Diagnose und praktische Gesichtspunkte sowie bewiesene Effekte der Beatmungstherapie und Hustenassistenz eingegangen.

\section{Neuromuskuläre Atemschwäche und schlafbezogene Atmungsstörungen $\nabla$}

Pathophysiologie Die fortschreitende neurogene Atrophie des Zwerchfells und der Atemhilfsmuskulatur führt zu einer Reduktion sowohl der Kraft als auch der Ausdauer betroffener Muskeln. Die verminderte Ventilation(sreserve) bedingt einen Anstieg des $\mathrm{CO}_{2}$-Partialdrucks im Blut, entweder tagsüber bei verstärkter körperlicher Aktivität oder nachts im Liegen, wenn aus mechanischen Gründen die Atemhilfsmuskulatur nicht effektiv eingesetzt werden kann und das Zwerchfell die Hauptlast der Atemarbeit trägt. Die schlafbezogene Hypoventilation ist die früheste Manifestation der muskulären Ateminsuffizienz [10]. Dies gilt insbesondere für den REM-Schlaf, in dem es physiologischerweise zu einer generalisierten Absenkung des Muskeltonus kommt. Ein kompensatorischer Anstieg der Atemfrequenz im Schlaf tritt nicht regelhaft auf und ist durch die verringerte Kapazität des Zwerchfells ohnehin limitiert. Der Anstieg des $\mathrm{pCO}_{2}$ beschränkt sich zu Anfang auf die REM-Schlaf-Phasen, dehnt sich mit zunehmender Zwerchfellparese auf die übrigen Schlafstadien aus und resultiert letztlich in einer Tageshyperkapnie, wenn die aufrechte Körperposition und der Einsatz der Atemhilfsmuskulatur zur Kompensation nicht mehr ausreichen.

Symptome Die schlafbezogene Hypoventilation bedingt Durchschlafstörungen, häufiges Erwachen aus dem Traum durch den $\mathrm{pCO}_{2}$-Anstieg im REM-Schlaf,

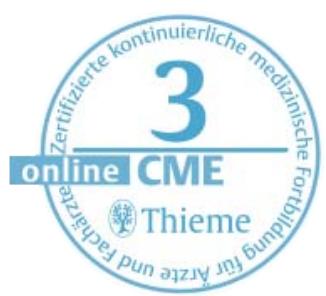


Abb. 1 Paradoxe Atmung. Normalerweise tritt das Zwerchfell bei der Inspiration in flacher Rückenlage soweit nach kaudal, dass es infolge des erhöhten intraabdominellen Drucks zu einer passiven Auslenkung der Bauchdecke nach oben kommt (a). Liegt eine Zwerchfellparese vor, wird die Bauchwandmuskulatur kompensatorisch eingesetzt, um das Zwerchfell zu unterstützen. Dadurch senkt sich die Bauchdecke bei der Inspiration ab (b).

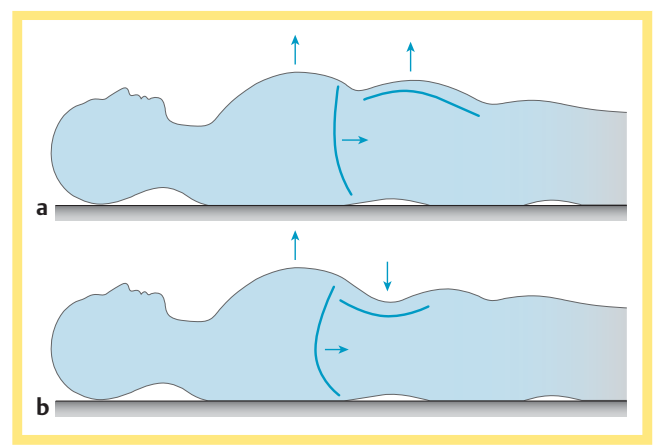

Tab. 1 Nicht pathologische Grenzwerte (lower limits of normal) für die Messung der Atemmuskelkraft mittels Spirometrie und Manometrie [14, 15].

\begin{tabular}{|c|c|c|c|}
\hline \multirow[t]{2}{*}{ Test } & \multirow[t]{2}{*}{ Methode } & \multicolumn{2}{|c|}{ nicht pathologischer Grenzwert } \\
\hline & & Frauen & Männer \\
\hline $\operatorname{MEP}\left(\mathrm{cm} \mathrm{H}_{2} \mathrm{O}\right)$ & Manometer & $\begin{array}{l}>70 \text { bzw. } \\
>95-(0,57 \times \text { Alter })\end{array}$ & $\begin{array}{l}>100 \text { bzw. } \\
>117-(0,83 \times \text { Alter })\end{array}$ \\
\hline PEF (I/min) & Peak-Flow-Meter & $>270$ & \\
\hline $\operatorname{MIP}\left(\mathrm{cm} \mathrm{H}_{2} \mathrm{O}\right)$ & Manometer & $\begin{array}{l}>60 \text { bzw. } \\
>62-(0,5 \times \text { Alter })\end{array}$ & $\begin{array}{l}>70 \text { bzw. } \\
>62-(0,15 \times \text { Alter })\end{array}$ \\
\hline $\operatorname{SNIP}\left(\mathrm{cm} \mathrm{H}_{2} \mathrm{O}\right)$ & Manometer & $>60$ & $>70$ \\
\hline VC & $\begin{array}{l}\text { Spirometer/Body- } \\
\text { plethysmografie }\end{array}$ & $\begin{array}{l}\text { aufrecht: }>80 \% \text { vom } \\
\text { liegend: }>80 \% \text { der in } \\
\text { gemessenen VC }\end{array}$ & echter Position \\
\hline
\end{tabular}

MEP: maximaler exspiratorischer Mundverschlussdruck, MIP: maximaler inspiratorischer Mundverschlussdruck, PEF: Peak Expiratory (Hustenspitzenstoß) Flow, SNIP: Sniff Nasal Inspiratory Pressure, VC: Vitalkapazität

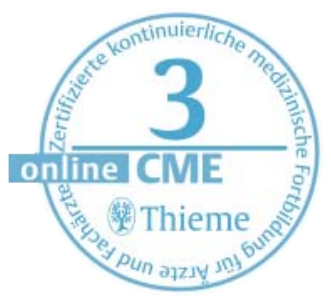

einen nicht erholsamen Nachtschlaf, morgendliche Kopfschmerzen, Benommenheit, Schwindel, Übelkeit und eine vermehrte Tagesschläfrigkeit. Patienten mit Atemmuskelschwäche berichten ferner über Luftnot bei Belastung oder später auch in Ruhe, körperliche Leistungsminderung und Fatigue, Orthopnoe und Hustenschwäche. In der klinischen Untersuchung können eine Tachypnoe (>14/min), eine Sprechdyspnoe und eine paradoxe Atmung ( $\bullet$ Abb. 1) in flacher Rückenlage auffällig sein.

Die alveoläre Hypoventilation manifestiert sich zuerst im (REM-)Schlaf.

\section{Diagnostik der Atemmuskelschwäche}

VC Die Messung der Vitalkapazität (VC) ist der gängigste Globaltest der in- und exspiratorischen Zwerchfellfunktion, auch wenn dadurch im engeren Sinne keine Kraftmessung erfolgt. Da die forcierte VC (FVC) insbesondere bei älteren Menschen aufgrund einer obstruktiven Komponente geringer ausfallen kann als die langsame VC (slow VC oder SVC), wird die Messung der SVC empfohlen. Diese kann mittels eines Hand- oder digitalen Spirometers oder im Rahmen der Bodyplethysmografie erfolgen; dabei ist die Verwendung einer Nasenklammer obligat. Für die Berechnung des Sollwerts werden Nomogramme oder Formeln herangezogen, die Alter, Geschlecht und Körpergröße berücksichtigen.

Hinweisend auf eine Atemmuskelschwäche ist eine VC $<80 \%$ des Sollwerts der eine Differenz von mehr als $20 \%$ zwischen der in aufrechter Position und der in flacher Rückenlage gemessenen VC. Eine VC unter $75 \%$ des Sollwerts ist bei ALS-Patienten ein unabhängiger Prädiktor für eine kurze Überlebenszeit [11]. Ferner lassen stark erniedrigte VC-Werte bei Patienten mit neuromuskulärer Ateminsuffizienz eine nächtliche Hyperkapnie (VC < 40\%) [12] oder sogar eine Tageshyperkapnie (VC $<25 \%$ ) [13] erwarten.

MIP/MEP Die Messung des maximalen in- und exspiratorischen Mundverschlussdrucks (MIP bzW. MEP) erfolgt mittels spezieller Manometer oder ebenfalls im Rahmen der Bodyplethysmografie. Auch hier muss eine Nasenklammer verwendet werden, um das Entweichen von Luft über die Nase zu verhindern. Bei Patienten mit ausgeprägter Mundschlussparese ist die Messung von VC, MIP und MEP oft nicht möglich, sodass entweder eine Mund-Nasen-Maske an das Spirometer bzw. Manometer adaptiert werden muss oder ersatzweise der maximale nasale Inspirationsdruck (sniff nasal inspiratory pressure, SNIP) gemessen werden sollte. Normwerte für die genannten Drücke sind in 0 Tab. 1 dargestellt. Eine Reduktion des MIP ist hinsichtlich einer nächtlichen Hypoventilation sensitiver als ein erniedrigter VCWert [16].

Peak Flow Die exspiratorische Muskelkraft kann nicht nur mittels MEP, sondern auch durch Husten in ein einfaches Peak-Flow-Meter gemessen werden (Nasenklemme!). Der Hustenspitzenstoß liegt bei Erwachsenen normalerweise oberhalb von 2701/min. Werte zwischen 160 und 270l/ min sprechen für eine klinisch relevante Hustenschwäche; unterhalb von 1601/min ist keine hinreichende bronchiale Sekretclearance mehr möglich [17].

Weitere Diagnosemethoden Unabhängig von der Mitarbeit des Patienten kann die Zwerchfellfunktion mittels motorischer Neurografie des N. phrenicus und bilateraler Magnetstimulation des N. phrenicus erfasst werden, letztere wird mit der Messung des Twitch-Munddrucks (Tw Pmo) oder des transdiaphragmalen Twitch-Drucks verbunden (Tw Pdi) [15]. Diese Messmethode steht allerdings routinemäßig nicht zur Verfügung, sodass bei Patienten, die z.B. aufgrund einer FTD für die Spiro- oder Manometrie nicht ausreichend kooperationsfähig sind, auf die Blutgasanalyse und die nächtliche Oxykapnometrie zurückgegriffen werden sollte (s. u.). Die Phrenicusneurografie hingegen ist leichter einsetzbar und insbesondere bei Patienten mit hochgradiger Mundschlussparese sinnvoll, die keine valide Spiromanometrie durchführen können. Die Amplitudenreduktion des motorischen Summenaktionspotenzials (MSAP) ist symmetrisch, sodass es ausreicht, eine Seite zu untersuchen (Norm- 
werte Tab.2). Erniedrigte MSAP-Amplituden korrelieren mit der FVC und dem SNIP, außerdem sind sie mit einer nächtlichen Hypoventilation und einer kürzeren Überlebenszeit assoziiert [19-22].

EMG Der Elektromyografie (EMG) sind sowohl das Zwerchfell als auch die akzessorischen Atemmuskeln, die interkostale und paravertebrale Muskulatur zugänglich. Die EMG tritt zur Diagnostik der Atemmuskelschwäche gegenüber den o.g. funktionellen Tests und der schlafmedizinischen Diagnostik aber in den Hintergrund. Im Zwerchfell-EMG weisen Zeichen der akuten und chronischen Denervation auf eine Beteiligung des N. phrenicus hin; die Nadelinsertion erfolgt am Unterrand des 8., 9. oder 10. Rippenknorpels [23]. Um die sehr seltene Komplikation eines Pneumothorax auf jeden Fall zu vermeiden, kann alternativ ein paravertebrales EMG in den Höhen C6 und Th5 durchgeführt werden, da neurogene Schädigungszeichen in diesem Bereich gut mit dem Zwerchfell-EMG und der VC korrelieren [24].

Die Kraft der Atemmuskulatur wird indirekt über die Vitalkapazität und direkt über die Messung des maximalen in- und exspiratorischen Drucks sowie des Hustenspitzenstoßes erfasst. Neurophysiologische Untersuchungsmethoden kommen ergänzend bei Patienten mit schwerer Mundschlussparese zur Anwendung.

\section{Schlafmedizinische Diagnostik}

Liegen Symptome einer schlafbezogenen Atmungsstörung vor und/oder unterschreiten VC, MIP oder SNIP den nicht pathologischen Grenzwert, ist eine weiterführende Diagnostik indiziert. Da die REMSchlaf-abhängige $\mathrm{CO}_{2}$-Retention die frühestmögliche Manifestation der alveolären Hypoventilation im Schlaf ist, wird vorrangig die kardiorespiratorische Polysomnografie (PSG) in Verbindung mit einer transkutanen Kapnografie empfohlen ( $\bullet$ Abb. 2). Neben der initial schlafstadienabhängigen Hypoventilation können eine obstruktive Schlafapnoe (Apnoe-Hypopnoe-Index $>5 / \mathrm{h} \mathrm{Ge-}$ samtschlafzeit), eine Fragmentierung des Nachtschlafs, ein verringerter Tiefschlafanteil und eine Reduktion des REM-Schlaf-Anteils auffällig sein.

Steht kein PSG-System zur Verfügung, kann alternativ eine Polygrafie erfolgen, dies jedoch auch immer in der Verbindung mit der Kapnometrie. Die alleinige nächtliche Pulsoxymetrie ist nicht ausreichend, da intermittierende Obstruktionen der oberen Atemwege nicht erfasst werden und über lange Zeit noch keine relevante Gasaustauschstörung feststellbar ist, auch wenn bereits eine Hypoventilation vorliegt. Eine frühmorgendliche (kapilläre) Blutgasanalyse (BGA) im Anschluss an die Diagnostiknacht ist generell zu empfehlen und muss als obligat angesehen werden, wenn kein nächtliches $\mathrm{CO}_{2}$-Monitoring erfolgt ist. Eine Basenabweichung $>4 \mathrm{mmol} / \mathrm{l}$ (Norm $\pm 3 \mathrm{mmol} / \mathrm{l}$ ) ist ein unabhängiger Prädiktor für eine nächtliche Hypoventilation [25]. Wichtig ist, dass eine später am Tag abgenommene BGA das Ausmaß der nächtlichen Hypoventilation oft nicht widerspiegelt, da eine kompensatorische Tachypnoe die Basenabweichung normalisieren und den $\mathrm{pCO}_{2}$ sogar absenken kann.

Die nächtliche Pulsoxymetrie allein ist diagnostisch nicht ausreichend. Polysomnografie oder Polygrafie sollten immer mit einer nächtlichen transkutanen Kapnometrie verbunden werden.

\section{Symptomatische Therapie}

\section{Nicht invasive Beatmung}

Indikation Gemäß der aktuellen Leitlinien ist bei Patienten mit neuromuskulärer Erkrankung und chronischer respiratorischer Insuffizienz die Einleitung einer nicht invasiven Beatmung (NIV) bei folgender Konstellation indiziert [26]:

1. Symptome einer schlafbezogenen Atmungsstörung oder Nachweis einer signifikanten muskulären Atemschwäche (VC $<50 \%$ des Sollwerts, $\mathrm{MIP}<60 \mathrm{~cm} \mathrm{H}_{2} \mathrm{O}$ ) und

2. Vorliegen mindestens eines der folgenden Kriterien:
a) Tageshyperkapnie $\left(\mathrm{pCO}_{2}>45 \mathrm{mmHg}\right)$
b) nächtliche Hyperkapnie $\left(\mathrm{tcCO}_{2}\right.$ oder $\mathrm{pCO}_{2}>50 \mathrm{mmHg}$ )
c) nächtlicher Anstieg des $\mathrm{pCO}_{2}$ oder $\mathrm{tcCO}_{2} \mathrm{um}$ mehr als $10 \mathrm{mmHg}$ über den Basal- oder Ta- geswert
d) rasche Reduktion der VC im Verlauf
Kontraindikation Gegen eine NIV sprechen
$\checkmark$ eine persistierende Enge der oberen Atem- wege,
> instabile Kiefer- oder Mittelgesichtsfrakturen,
$\checkmark$ eine unkontrollierbare bronchiale Hypersekre- tion,
$\checkmark$ ein trotz technischer Hilfsmittel dauerhaft in- effektiver Hustenstoß und
> eine Konstellation, in welcher der Patient selbst oder seine Hilfspersonen zur Anwendung der NIV nicht in der Lage sind.

Tab. 2 Normwerte für die motorische Neurografie des N. phrenicus. Stimulationsort: $3 \mathrm{~cm}$ oberhalb der Klavikula dorsal des M. sternocleidomastoideus, Ableitort: 8. Interkostalraum in der vorderen Axillarlinie [18].

\begin{tabular}{|lcccc|}
\hline & & $\begin{array}{l}\text { Mittelwert } \\
\text { (SD) }\end{array}$ & $\begin{array}{l}\text { unterer } \\
\text { Grenzwert }\end{array}$ & $\begin{array}{c}\text { oberer } \\
\text { Grenzwert }\end{array}$ \\
\hline Latenz $(\mathrm{ms})$ & Inspiration & $6,6(0,7)$ & 5,2 & 7,9 \\
\cline { 2 - 5 } & Exspiration & $6,6(0,7)$ & 5,3 & 7,9 \\
\hline Amplitude $(\mathrm{mV})$ & Inspiration & $1,0(0,3)$ & 0,46 & 1,5 \\
\hline Potenzialdauer $(\mathrm{ms})$ & Exspiration & $0,7(0,2)$ & 0,33 & 1,1 \\
\hline & Inspiration & $15,0(3,1)$ & 8,7 & 21,3 \\
\hline & Exspiration & $21,0(3,3)$ & 16,1 & 28,3 \\
\hline
\end{tabular}




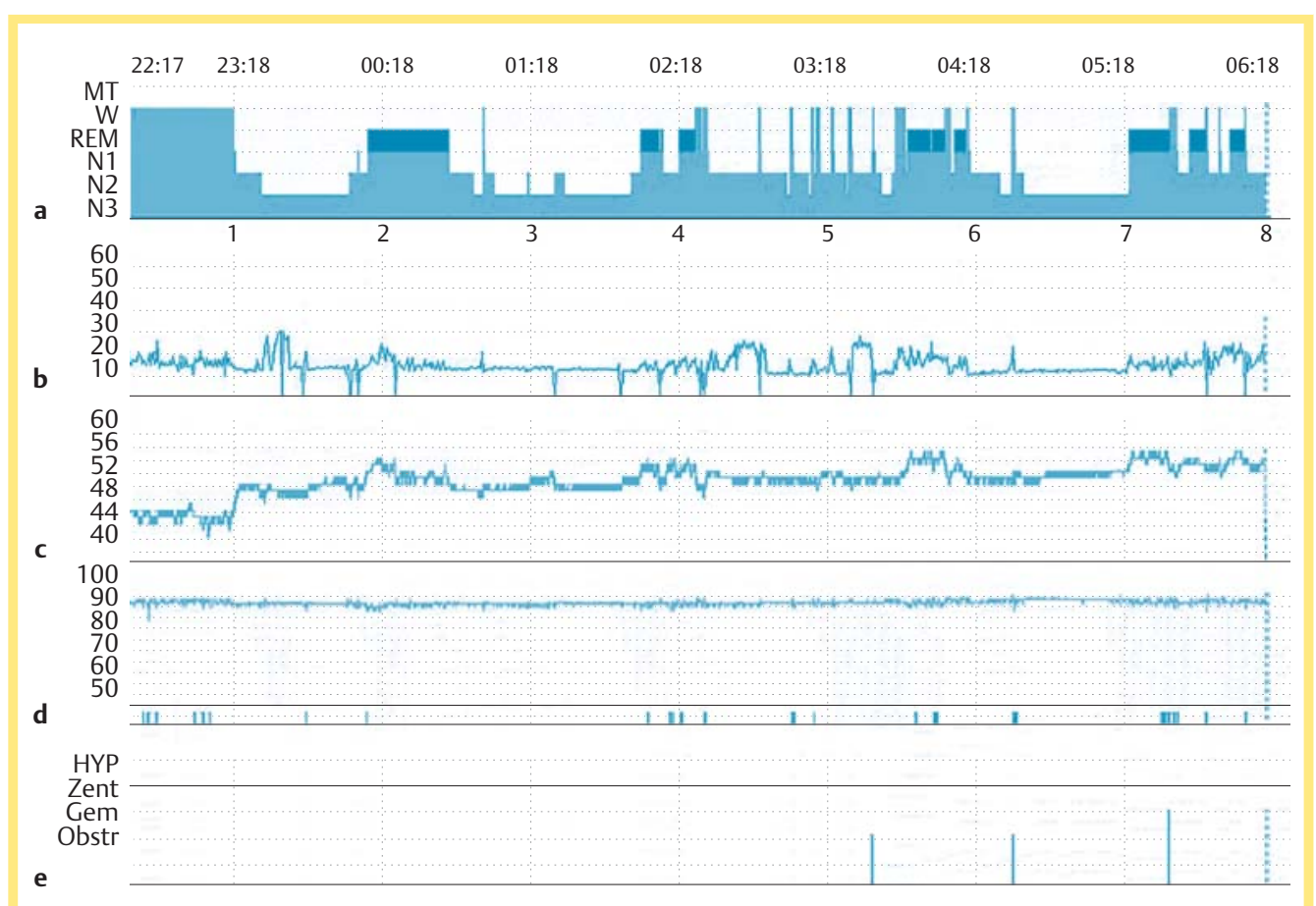

Abb.2 Polysomnografiebefund eines Patienten mit ALS, morgendlichen Kopfschmerzen, einer SVC von $61 \%$ des Sollwerts und einem MIP von $49 \mathrm{~cm} \mathrm{H} \mathrm{H}_{2} \mathrm{O}$, zeitsynchrone Darstellung von Hypnogramm (a), Atemfrequenz (b), transkutan gemessenem $\mathrm{CO}_{2}$ (c), Sauerstoffsättigung (d) und Apnoen (e). Das gesamte Schlafprofil und insbesondere die REM-Schlaf-Phasen (schwarze Balken) in der 2. Nachthälfte sind fragmentiert. Nach dem Einschlafen steigt der transkutane $\mathrm{CO}_{2}$-Wert $\left(\mathrm{tc} \mathrm{CO}_{2}\right)$ an und zeigt über den weiteren Verlauf der Nacht eine weiter ansteigende Tendenz (=Erschöpfung der Atemmuskulatur). Während des REMSchlafs nimmt die Hyperkapnie trotz Erhöhung der Atemfrequenz zu (=Verstärkung der Hypoventilation im REM-Schlaf). Ereignisse, welche die Kritierien einer Apnoe oder Hypopnoe erfüllen, treten nur ganz vereinzelt auf, entsprechend zeigt die Sauerstoffsättigung über die gesamte Nacht unauffällige Werte. Ohne die Kapnometrie wäre die Hypoventilation der Diagnostik entgangen (MT: Movement Time, W: Wake, REM: REM-Schlaf, N1: Einschlafen, N2: Leichtschlaf, N3: Tiefschlaf).

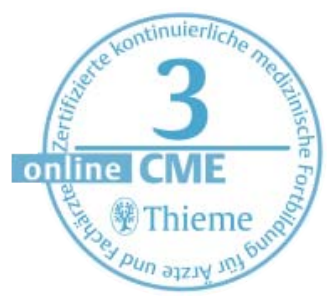

Ferner sind eine schwerste Dysphagie und die primäre Notwendigkeit einer 24-h-Beatmung relative Kontraindikationen, sodass bei entsprechendem Patientenwunsch der invasiven mechanischen Beatmung (IMV) der Vorzug gegeben werden sollte.

Durchführung Die NIV sollte grundsätzlich unter stationären Bedingungen und unter poly(somno)grafischer und kapnometrischer Kontrolle über mehrere Nächte eingeleitet werden. Patienten und Angehörige müssen ausführlich über die Therapie aufgeklärt und in deren Handhabung eingewiesen werden. Beatmungsgerät, Maske und Geräteeinstellungen werden entsprechend des schlafmedizinischen Ausgangsbefundes und der individuellen Bedürfnisse von geschultem Personal angepasst. Beatmungsmodus der Wahl ist eine druckkontrollierte, zeit- und volumengesteuerte Bilevel-Therapie mit der Möglichkeit zur bedarfsweisen Triggerung von zusätzlichen Atemzügen durch den Patienten. Die Geräteparameter (endexspiratorischer und inspiratorischer Druck, mandatorische Frequenz, Tidalvolumen, Inspirationszeit) werden so gewählt, dass die Hypoventilation adäquat ausgeglichen ist und etwaige Obstruktionen der oberen Atemwege beseitigt werden.

Gerätewahl Wenn möglich, sollte aus Gründen der Tolerabilität initial eine Nasenmaske gewählt werden. Eine Mund-Nasen-Maske ist erforderlich, wenn eine relevante Mundschlussparese besteht, allerdings können darunter vermehrt obstruktive Hypopnoen auftreten, sodass der endexspiratorische Druck erhöht oder auf eine Nasenmaske mit Kinnband gewechselt werden muss [27]. Sofern keine Lungenparenchymerkrankung bzw. Gasaustauschstörung vorliegt, ist eine ergänzende Sauerstoffvorlage nicht indiziert. Eine Warmluftbefeuchtung ist in aller Regel erforderlich. Übersteigt die tägliche Beatmungsdauer 16 Stunden, muss ein zweites Beatmungsgerät verordnet werden. Geräte mit leistungskräftigem Akku sind zu bevorzugen, um die Mobilität am Tag bestmöglich zu erhalten.

Qualifiziertes Monitoring, schrittweise Titration der Beatmungsmodalitäten, Patientenedukation und ausreichend Zeit sind Voraussetzungen einer erfolgreichen Therapieeinleitung.

Effekte Die Empfehlung zur nicht invasiven Beatmung ist Bestandteil aller internationalen Leitlinien zur Therapie der ALS [28]. Zahlreiche Studien belegen eine Verbesserung der gesundheitsbezogenen Lebensqualität, der subjektiven Schlafqualität und der Fatigue durch die NIV [29, 30]. Ferner reduziert die NIV Symptome wie Tagesschläfrig- 
keit, Dyspnoe und Depressivität [31]. Die objektive Verbesserung und Stabilisierung des Schlafprofils und der nächtlichen Ventilation konnte sowohl kurzfristig als auch im längeren zeitlichen Verlauf nachgewiesen werden [32, 33]. Hinsichtlich der Zwerchfellfunktion und der Lebenserwartung unter NIV belegen mehrere Studien den positiven Effekt einer regelmäßigen und ausreichenden Gerätenutzung von zu Anfang mindestens 4 Stunden pro Nacht [34-37]. Eine einzige kontrollierte randomisierte Studie mit 41 Patienten zeigte in der mit NIV behandelten Gruppe von Patienten mit spinaler ALS eine deutlich bessere Lebensqualität sowie eine mittlere Überlebenszeitverlängerung von 216 Tagen im Vergleich zur Kontrollgruppe [29]. Damit ist die NIV der medikamentösen Therapie mit Riluzol eindeutig überlegen (ca. 3 Monate Überlebensvorteil $[38,39])$.

NIV verlängert bei Patienten mit spinaler ALS die mittlere Überlebenszeit deutlicher als Riluzol. Ferner verbessert die NIV für Patienten mit spinaler ALS und (eingeschränkt) auch für Patienten mit bulbärem Verlaufstyp die Lebensqualität.

\section{Invasive mechanische Beatmung}

Indikation Unter der Voraussetzung eines entsprechenden Patientenwillens ist die IMV unter folgenden Umständen medizinisch indiziert:

- unzureichende Behandlung der Hypoventilation durch eine maximal ausgereizte NIV

Intolerabilität gegenüber der NIV

- Kontraindikationen gegen eine NIV

NIV über $>20 \mathrm{~h}$ pro Tag

- schwerste Dysphagie und Aspirationsgefahr

- Versagen nicht invasiver Methoden des Sekretmanagements

gescheiterter NIV-Versuch nach passagerer invasiver Tubusbeatmung

Vor einem Wechsel von der NIV auf die IMV sollte sorgfältig geprüft werden, ob nicht durch Optimierung der Geräteeinstellungen, der Maskenversorgung oder des Sekretmanagements eine IMV doch noch umgangen werden kann.

Durchführung Ist die Tracheotomie nicht zu vermeiden und wird sie gewünscht, ist die operative Tracheostomaanlage der Bougierungstracheotomie immer vorzuziehen. Neben der weiterhin druckkontrollierten, zeit- und volumengesteuerten Beatmung müssen eine intensivierte Luftbefeuchtung und ein invasives Sekretmanagement etabliert werden. Für artikulationsfähige Patienten stehen spezielle Sprechkanülen zur Verfügung. Pflegende Angehörige sind ausführlich zu schulen und es ist ratsam, einen häuslichen 24-h-Pflegedienst hinzuziehen, der über zertifizierte Expertise in der außerklinischen Beatmung verfügt. Die Versorgung mit 2 Beatmungsgeräten ist obligat.

Verlaufskontrolle Beatmungsmedizinische Kontrolluntersuchungen mit Polygrafie und nächtli- chem $\mathrm{CO}_{2}$-Monitoring sollten bei Patienten mit NIV oder IMV 2-4-mal jährlich erfolgen, bei entsprechender Notwendigkeit auch in kürzeren Abständen, um die Geräteparameter an die fortschreitende Atemmuskelschwäche anzupassen. Ferner müssen betroffene Patienten und Familien die Möglichkeit haben, jederzeit Kontakt zur versorgenden beatmungsmedizinischen Krankenhausabteilung aufzunehmen.

Die invasive Langzeitbeatmung über eine Trachealkanüle ist eine therapeutische Option, wenn die NIV nicht initiiert oder fortgesetzt werden kann und zugleich ein entsprechender Patientenwille vorhanden ist.

\section{Zwerchfellstimulation}

Auf der Grundlage mehrerer Studien wurde 2011 die elektrische Zwerchfellstimulation von der US-Arzneimittelbehörde im Rahmen einer Ausnahmeregelung für Patienten mit ALS zugelassen [40]. Bei dieser Methode werden 4 Stimulationselektroden laparaskopisch ins Zwerchfell implantiert. Notwendige Voraussetzungen für diesen Eingriff sind eine VC von mindestens $50 \%$ des Sollwerts und erhaltene Reizantworten in der Phrenicusneurografie. Die Zwerchfellstimulation kann bei bereits vorhandener Hypoventilation mit der NIV kombiniert werden. Bereits publizierte Ergebnisse von Beobachtungsstudien legen einen verzögernden Einfluss auf die Abnahme der VC im Krankheitsverlauf nahe [41]. In der bisher einzigen randomisierten und kontrollierten Studie (DiPALS-Studie) wurden Patienten mit klinisch manifester Atemmuskelschwäche entweder mit NIV allein oder mit NIV und Zwerchfellstimulation behandelt. Nachdem Interimsanalysen eine geringere Überlebenszeit der Patienten mit Zwerchfellschrittmacher nahegelegt hatten, erfolgte ein vorzeitiger Rekrutierungsstopp. Die aktuell publizierten Ergebnisse der Endauswertung zeigen einen deutlichen Unterschied in der Überlebenszeit zugunsten der nicht operierten Gruppe (22,8 gegenüber 11,1 Monate [42]). Daraus resultiert, dass die Schrittmachertherapie dann nicht zum Einsatz kommen sollte, wenn bereits eine relevante Zwerchfellparese mit Indikation zur NIV vorliegt. 


\section{Fazit: Beatmung}

In der Summe ist die NIV eine symptomorientierte Therapieoption mit dem realistischen Potenzial einer Verbesserung von Lebensqualität und Überlebenszeit, die gegenüber allen Patienten mit ALS frühzeitig angesprochen werden sollte, auch wenn noch keine klinischen Hinweise auf eine muskuläre Ateminsuffizienz vorliegen. Regelmäßige Verlaufsuntersuchungen einschließlich schlafmedizinischer Anamnese, Spirometrie und Manometrie sind die Voraussetzung dafür, die Indikation zu einer weiterführenden schlafmedizinischen Diagnostik frühzeitig stellen zu können und bei Vorliegen eines Hypoventilationssyndroms auch früh mit der NIV zu beginnen. Dies erleichtert vielen Patienten die Adaptation an die Therapie und hat nach aktueller Studienlage zusätzliche Bedeutung für die Prognose der Erkrankung: Frühe nicht invasive Beatmung ist mit einem längeren Überleben assoziiert [43]. Unter medizinischen und ethischen Gesichtspunkten sollte die frühzeitige NIV also unmissverständlich empfohlen werden. Allerdings kann es hilfreich sein, Patienten deutlich zu machen, dass im Sinne der Entscheidungsautonomie am Lebensende jede Form der Beatmung auch wieder beendet bzw. ausgesetzt werden kann, wenn dies dem erklärten Patientenwillen entspricht und eine qualifizierte palliativmedizinische Begleitung gewährleistet ist. Die Entscheidung für oder gegen die Tracheotomie sollte idealerweise in einer Patientenverfügung vorweggenommen sein und richtet sich im Einzelfall nach höchst individuellen Kriterien, die dem spezifischen klinischen Zustand, der Lebens- und sozialen Situation sowie den persönlichen Einstellungen des Patienten entspringen.

\section{Sekretmanagement}

Hustenschwäche Durch die fortschreitende Lähmung des Zwerchfells und der Atemhilfsmuskulatur entwickeln Patienten mit ALS nicht nur eine inspiratorische Ateminsuffizienz, sondern auch eine Hustenschwäche, die erhebliche Bedeutung für Lebensqualität und -erwartung hat. Insbesondere bei Patienten mit bulbärer ALS kommt erschwerend hinzu, dass die Dysphagie mit einem hohen Aspirationsrisiko einhergeht und die Insuffizienz des Glottisschlusses den Hustenstoß gänzlich ineffektiv machen kann. Für dysphage Patienten besteht ein relevantes Risiko, sowohl größere Speisebrocken zu aspirieren als auch rezidivierend schwere Infekte der unteren Atemwege zu erleiden. Neben einer adäquaten Anpassung der Ernährung und der Nahrungskonsistenz bis hin zur Anlage einer PEG-Sonde dient daher die effektive Unterstützung des Hustenstoßes der Prophylaxe des Bolustodes und der Aspirationspneumonie; ferner ist die Hustenassistenz während einer akuten respiratorischen Verschlechterung therapeutisch essenziell.
Indikation Eine Unterstützung des Hustens ist indiziert, wenn der Hustenspitzenstoß 2701/min unterschreitet und/oder die periphere Sauerstoffsättigung unter $95 \%$ bzw. $2-3 \%$ unterhalb des individuellen Ausgangswerts liegt [44].

Manuelle Methoden Maßnahmen der manuellen Hustenassistenz umfassen posturale Drainage und externe Bauchpresse, die von geschulten Physiound Atemtherapeuten eingesetzt werden, aber auch von Angehörigen erlernt werden können. Da der beim Husten erreichte exspiratorische Flow wesentlich vom Inspirationsvolumen abhängt, kann dieses durch wiederholtes Einatmen ohne Exspiration oder durch Air Stacking mithilfe eines Beatmungsbeutels mit Mundstück gesteigert werden [45]. Diese Methode ist auch bei beatmeten Patienten über die Maske oder die Trachealkanüle realisierbar.

Technische Methoden Technische Hilfsmittel zur Hustenassistenz sind indiziert, wenn manuelle Methoden in Kombination mit Air Stacking nicht ausreichend sind oder nicht angewendet werden können und wenn der Hustenspitzenstoß 1601/ min unterschreitet. Geräte zur Insufflation/Exsufflation $(I / E)$ verbinden einen tiefen maschinellen Inspirationshub mit einem plötzlichen Sog, der einen hohen exspiratorischen Flow erzeugt und damit den Hustenstoß unterstützt oder die bronchiale Clearance ganz ersetzt. I/E-Geräte reduzieren das Risiko pulmonaler Infekte deutlich und senken die Hospitalisierungsrate [46, 47]. Eine erste Vergleichsstudie zwischen Air Stacking und Insufflation/Exsufflation lieferte aus methodischen Gründen keine statistisch signifikanten Ergebnisse [48]. Im Fall großer oder zäher Sekretmengen, die sich trotz ausreichender Flüssigkeitszufuhr nicht vollständig expektorieren lassen, ist die Kombination mit einem Gerät zur hochfrequenten Brustwandoszillation (high frequency chest wall oscillation, HFCWO) oder mit einer intermittierend angewandten intrapulmonalen perkussiven Ventilation (IPV) anzuraten. Beide Methoden verbessern die mukoziliare Clearance und befördern Sekret aus der Lungenperipherie in die großen Atemwege, von wo es mittels eines I/E- oder Absauggeräts entfernt werden kann [49]. Kontraindikationen gegen die Anwendung der Insufflation/Exsufflation und der HFCWO umfassen Thoraxinstabilität, Lungenemphysem, schwere Herzinsuffizienz, Pneumothorax, Pneumomediastinum und schweres Asthma bronchiale.

Wirkstoffe Zur Verringerung von bronchialer Hypersekretion und Pseudohypersalivation können anticholinerg wirksame Substanzen wie AtropinTropfen sublingual, N-Butylscopolamin (Pflaster) oder retardiertes Amitriptylin zum Einsatz kommen. Alternativ oder ergänzend steht zur Reduktion des Speichelflusses die Injektion von Botulinumtoxin A in die Gll. parotides und die Gll. submandibulares zur Verfügung. 


\section{Fazit Sekretmanagement}

Ein vorausschauendes und qualifiziertes Sekretmanagement ist nicht nur für Patienten mit bulbärer ALS von vitaler Bedeutung. Dieses umfasst Sekretreduktion, manuelle Hustenhilfen und, wenn indiziert, apparative Verfahren zur Mobilisation und Expektoration von Sekret. Beatmung und Sekretmanagement sind eng miteinander verbunden und sollten immer gleichzeitig bedacht und eingeleitet werden.

\section{Zusammenfassung \\ $\nabla$}

Die Symptome und Folgen der muskulären Ateminsuffizienz tragen bei der amyotrophen Lateralsklerose maßgeblich zur Morbidität und Mortalität der Erkrankung bei und beeinträchtigen nachhaltig die Lebensqualität. Während initial Symptome und Zeichen der schlafbezogenen Atmungsstörung im Vordergrund stehen, kommt es im Verlauf zu chronischem hyperkapnischem Atemversagen und zu einer klinisch hoch relevanten Hustenschwäche mit Sekretverhalt, fehlendem Schutz vor Aspiration und erhöhtem Infektrisiko. Die frühzeitige Erkennung und adäquate Therapie atmungsassoziierter Symptome ist für die Lebensqualität und die Prognose der betroffenen Patienten entscheidend. Dieser Übersichtsartikel fasst den gegenwärtigen Stand der diagnostischen und therapeutischen Möglichkeiten zusammen und gibt praxisrelevante Empfehlungen zum Management der in- und exspiratorischen Atemmuskelschwäche.

Interessenkonflikt: MB und PY haben von Heinen + Löwenstein, Bad Ems, Referentenhonorare und Mittel zur Durchführung wissenschaftlicher Projekte erhalten.

\section{Literatur}

1 Chio A, Logroscino G, Traynor BJ et al. Global epidemiology of amyotrophic lateral sclerosis: a systematic review of the published literature. Neuroepidemiology 2013; 41: 118-130

2 Mehta P, Antao V, Kaye $W$ et al. Prevalence of amyotrophic lateral sclerosis - United States, 2010-2011. MMWR Surveill Summ 2014; 63 (Suppl 7): 1-14

3 Tsai CP, Wang KC, Hwang CS et al. Incidence, prevalence, and medical expenditures of classical amyotrophic lateral sclerosis in Taiwan, 1999-2008. Journal of the Formosan Medical Association 2015; 114: 612-619

4 Shoesmith CL, Findlater K, Rowe A et al. Prognosis of amyotrophic lateral sclerosis with respiratory onset. Journal of neurology, neurosurgery, and psychiatry 2007; 78: 629-631

5 Gil J, Funalot B, Verschueren A et al. Causes of death amongst French patients with amyotrophic lateral sclerosis: a prospective study. European journal of neurology: the official journal of the European Federation of Neurological Societies 2008; 15: 1245 - 1251
6 Czaplinski A, Yen AA, Simpson EP et al. Slower disease progression and prolonged survival in contemporary patients with amyotrophic lateral sclerosis: is the natural history of amyotrophic lateral sclerosis changing? Archives of neurology 2006; 63: 1139-1143

7 O'toole O, Traynor BJ, Brennan P et al. Epidemiology and clinical features of amyotrophic lateral sclerosis in Ireland between 1995 and 2004. Journal of neurology, neurosurgery, and psychiatry 2008; 79: 30-32

8 Zoccolella S, Beghi E, Palagano G et al. ALS multidisciplinary clinic and survival. Results from a population-based study in Southern Italy. Journal of neurology 2007; 254: $1107-1112$

9 Engel GL. The need for a new medical model: a challenge for biomedicine. Science 1977; 196: 129-136

10 Perrin C, Unterborn JN, Ambrosio CD et al. Pulmonary complications of chronic neuromuscular diseases and their management. Muscle \& nerve 2004; 29: 5-27

11 Czaplinski A, Yen AA, Appel SH. Forced vital capacity (FVC) as an indicator of survival and disease progression in an ALS clinic population. Journal of neurology, neurosurgery, and psychiatry 2006; 77: 390-392

12 Ragette R, Mellies U, Schwake $C$ et al. Patterns and predictors of sleep disordered breathing in primary myopathies. Thorax 2002; 57: 724-728

13 Toussaint M, Steens M, Soudon P. Lung function accurately predicts hypercapnia in patients with Duchenne muscular dystrophy. Chest 2007; 131: 368-375

14 Evans JA, Whitelaw WA. The assessment of maximal respiratory mouth pressures in adults. Respiratory care 2009; 54: 1348 - 1359

15 Kabitz HJ, Walterspacher S, Mellies U et al. Messung der Atemmuskelfunktion. Empfehlungen der Deutschen Atemwegsliga e.V. in der Deutschen Gesellschaft für Pneumologie und Beatmungsmedizin (DGP). München, Orlando: Dustri-Verlag Dr. Karl Feistle; 2014

16 Jackson CE, Rosenfeld J, Moore DH et al. A preliminary evaluation of a prospective study of pulmonary function studies and symptoms of hypoventilation in ALS/ MND patients. Journal of the neurological sciences 2001; 191: 75-78

17 Kang SW, Bach JR. Maximum insufflation capacity: vital capacity and cough flows in neuromuscular disease. American journal of physical medicine \& rehabilitation/Association of Academic Physiatrists 2000; 79: 222-227

18 Preston DC, Shapiro BE. Electromyography and Neuromuscular Disorders: Clinical-Electrophysiologic Correlations. 3rd ed. Elsevier; 2014

19 Pinto S, De Carvalho M. Symmetry of phrenic nerve motor response in amyotrophic lateral sclerosis. Muscle \& nerve 2010; 42: 822 - 825

20 Pinto S, Geraldes $R$, Vaz $N$ et al. Changes of the phrenic nerve motor response in amyotrophic lateral sclerosis: longitudinal study. Clinical neurophysiology 2009; 120: 2082-2085

21 Pinto S, Pinto A, De Carvalho M. Phrenic nerve studies predict survival in amyotrophic lateral sclerosis. Clinical neurophysiology 2012; 123: 2454-2459

22 Pinto S, Turkman A, Pinto A et al. Predicting respiratory insufficiency in amyotrophic lateral sclerosis: the role of phrenic nerve studies. Clinical neurophysiology 2009; 120: 941 -946

23 Saadeh PB, Crisafulli CF, Sosner J et al. Needle electromyography of the diaphragm: a new technique. Muscle \& nerve 1993; 16: 15-20

24 De Carvalho M, Pinto S, Swash M. Association of paraspinal and diaphragm denervation in ALS. Amyotrophic lateral sclerosis 2010; 11: 63-66

25 Hukins CA, Hillman DR. Daytime predictors of sleep hypoventilation in Duchenne muscular dystrophy. American journal of respiratory and critical care medicine 2000; 161: $166-170$

26 Windisch W, Brambring J, Budweiser S et al. [Non-invasive and invasive mechanical ventilation for treatment

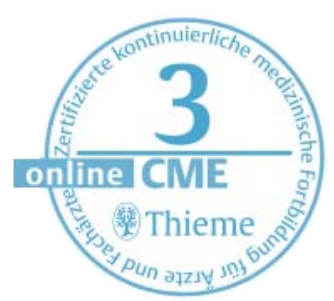


of chronic respiratory failure. S2-Guidelines published by the German Medical Association of Pneumology and Ventilatory Support]. Pneumologie 2010; 64: 207-240

27 Vrijsen B, Buyse B, Belge $C$ et al. Upper airway obstruction during noninvasive ventilation induced by the use of an oronasal mask. JCSM 2014; 10: 1033 1035

28 Miller RG, Jackson CE, Kasarskis EJ et al. Practice parameter update: the care of the patient with amyotrophic lateral sclerosis: drug, nutritional, and respiratory therapies (an evidence-based review): report of the Quality Standards Subcommittee of the American Academy of Neurology. Neurology 2009; 73: $1218-1226$

29 Bourke SC, Tomlinson M, Williams TL et al. Effects of non-invasive ventilation on survival and quality of life in patients with amyotrophic lateral sclerosis: a randomised controlled trial. The Lancet. Neurology 2006; 5: 140-147

30 Lyall RA, Donaldson N, Fleming $T$ et al. A prospective study of quality of life in ALS patients treated with noninvasive ventilation. Neurology 2001; 57: 153 156

31 Butz M, Wollinsky KH, Wiedemuth-Catrinescu U et al. Longitudinal effects of noninvasive positive-pressure ventilation in patients with amyotrophic lateral sclerosis. American journal of physical medicine \& rehabilitation 2003; 82: 597-604

32 Boentert M, Brenscheidt I, Glatz C et al. Effects of noninvasive ventilation on objective sleep and nocturnal respiration in patients with amyotrophic lateral sclerosis. Journal of neurology 2015; 262: 2073-2082

33 Vrijsen B, Buyse B, Belge Cet al. Noninvasive ventilation improves sleep in amyotrophic lateral sclerosis: a prospective polysomnographic study. JCSM 2015; 11: 559-566

34 Aboussouan LS, Khan SU, Meeker DP et al. Effect of noninvasive positive-pressure ventilation on survival in amyotrophic lateral sclerosis. Annals of internal medicine 1997; 127: 450-453

35 Kleopa KA, Sherman M, Neal B et al. Bipap improves survival and rate of pulmonary function decline in patients with ALS. Journal of the neurological sciences 1999; 164: $82-88$

36 Mustfa N, Walsh E, Bryant $V$ et al. The effect of noninvasive ventilation on ALS patients and their caregivers. Neurology 2006; 66: 1211 - 1217

37 Pinto AC, Evangelista T, Carvalho $M$ et al. Respiratory assistance with a non-invasive ventilator (Bipap) in MND/ALS patients: survival rates in a controlled trial.
Journal of the neurological sciences 1995; 129 (Suppl): $19-26$

38 Bensimon G, Lacomblez L, Meininger V. A controlled trial of riluzole in amyotrophic lateral sclerosis. ALS/ Riluzole Study Group. The New England journal of medicine 1994; 330: 585-591

39 Lacomblez L, Bensimon G, Leigh PN et al. A confirmatory dose-ranging study of riluzole in ALS. ALS/Riluzole Study Group-II. Neurology 1996; 47: S242 - 250

40 http://www.fda.gov/MedicalDevices/Productsand MedicalProcedures/DeviceApprovalsandClearances/ Recently-ApprovedDevices/ucm278684.htm

41 Onders RP, Elmo M, Kaplan C et al. Final analysis of the pilot trial of diaphragm pacing in amyotrophic lateral sclerosis with long-term follow-up: diaphragm pacing positively affects diaphragm respiration. American journal of surgery 2014; 207: 393-397; discussion 397

42 DiPALS Writing Committee, DiPALS Study Group Collaborators. Safety and efficacy of diaphragm pacing in patients with respiratory insufficiency due to amyotrophic lateral sclerosis (DiPALS): a multicentre, open-label, randomised controlled trial. The Lancet Neurology 2015; 14: 883-892

43 Carratu P, Spicuzza L, Cassano A et al. Early treatment with noninvasive positive pressure ventilation prolongs survival in Amyotrophic Lateral Sclerosis patients with nocturnal respiratory insufficiency. Orphanet journal of rare diseases 2009; 4: 10

44 Windisch W, Walterspacher S, Siemon K et al. Guidelines for non-invasive and invasive mechanical ventilation for treatment of chronic respiratory failure. Published by the German Society for Pneumology (DGP). Pneumologie 2010; 64: 640-652

45 Pryor JA. Physiotherapy for airway clearance in adults. The European respiratory journal 1999; 14: $1418-1424$

46 Bach JR, Ishikawa Y, Kim H. Prevention of pulmonary morbidity for patients with Duchenne muscular dystrophy. Chest 1997; 112: 1024-1028

47 Tzeng AC, Bach JR. Prevention of pulmonary morbidity for patients with neuromuscular disease. Chest 2000; 118: 1390 - 1396

48 Rafiq MK, Bradburn M, Proctor AR et al. A preliminary randomized trial of the mechanical insufflator-exsufflator versus breath-stacking technique in patients with amyotrophic lateral sclerosis. Amyotrophic lateral sclerosis \& frontotemporal degeneration 2015; DOI: 10.3109/21678421.2015.1051992

49 Chatburn RL. High-frequency assisted airway clearance. Respiratory care 2007; 52: 1224-1235; discussion 1235-1227

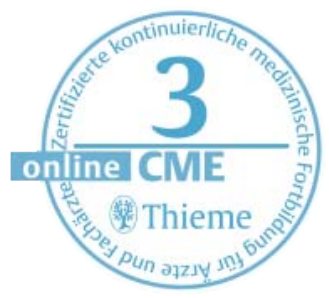




\section{CME-Fragen Beatmung und Sekretmanagement bei amyotropher Lateralsklerose ${ }^{1}$}

\section{Welche der folgenden Aussagen ist zutreffend?}

A Nur wenige Patienten mit ALS entwickeln im Verlauf der Erkrankung eine Schwäche der Atemmuskulatur.

B Das Zwerchfell ist in der Regel nur einseitig in die Erkrankung einbezogen, so dass die Atemmuskelschwäche über lange Zeit kompensiert werden kann.

C Es ist ratsam, Patienten mit ALS frühzeitig darüber aufzuklären, dass im Verlauf der Erkrankung eine Schwäche der Atemmuskulatur wahrscheinlich auftreten und sich die Notwendigkeit einer atmungsunterstützenden Therapie ergeben wird.

D Patienten mit ALS versterben in der Regel nicht an den Folgen der Atemmuskelschwäche.

E Bei Patienten mit primär bulbärem Verlaufstyp kommt eine Beteiligung der Atemmuskulatur nur sehr selten vor.

F Die motorische Neurographie des N. phrenicus ist eine nicht-invasive und objektive Methode, um die Funktion des Zwerchfells zu untersuchen.

Welche der folgenden Aussagen ist richtig?

A Kopfschmerzen infolge einer hyperkapnischen respiratorischen Insuffizienz treten in der Regel abends auf.

B Aufgrund der physiologischen Absenkung des Muskeltonus im REM-Schlaf tritt eine alveoläre Hypoventilation mit $\mathrm{CO}_{2}$-Retention zuallererst in diesem Schlafstadium auf.

C Die reduzierte körperliche Leistungsfähigkeit von Patienten mit ALS kann allein mit den Extremitätenparesen hinreichend erklärt werden.

D Patienten mit ALS, die über nächtliche Luftnot und Angst berichten, sollten mit einem langwirksamen Benzodiazepin am Abend behandelt werden.

E Solange Patienten nicht über Einschlafattacken am Tag oder lautes Schnarchen in der Nacht berichten, ist keine weiterführende schlafmedizinische Diagnostik erforderlich.

\section{Welche der folgenden Aussagen ist falsch?}

A Die nächtliche Kapnometrie ist in den Indikationskriterien für die Einleitung der nicht-invasiven Beatmung berücksichtigt.

B Es gibt Evidenz dafür, dass ein früher Beginn der nicht-invasiven Beatmung bei ALS-Patienten mit einer längeren Überlebenszeit assoziiert ist.

c Hinsichtlich der Verlängerung des Überlebens ist die nichtinvasive Beatmung bei Patienten mit spinaler ALS der medikamentösen Therapie mit Riluzol weit überlegen.

D Die nicht-invasive Beatmung kann die klinischen Symptome der schlafbezogenen Hypoventilation lindern und auf diese Weise die Lebensqualität verbessern.

E Für Patienten mit bulbärer ALS und Schluckstörung ist die nicht-invasive Beatmung kontraindiziert.

\section{4}

A Die Entscheidung zur Tracheotomie sollte in Abwägung der medizinischen Situation und des Patientenwillens individuell getroffen werden.

B Es ist zu empfehlen, dass Patienten ihre Entscheidung für oder gegen eine Tracheotomie in einer Patientenverfügung niederlegen.

C Nach Anlage eines Tracheostomas besteht automatisch die Notwendigkeit einer 24-h-Beatmung.

D Für mobile Patienten mit hochgradiger Dysphagie und Anarthrie kann die frühzeitige Tracheotomie eine geeignete therapeutische Maßnahme darstellen.

E Die invasive Langzeitbeatmung sollte über ein operativ angelegtes Tracheostoma erfolgen.

\section{5}

A Techniken der manuellen Hustenassistenz können auch von pflegenden Angehörigen erlernt werden.

B Beim sogenannten Air Stacking wird durch wiederholte aktive oder passive Inspiration die Vitalkapazität vergrößert, um den nachfolgenden Hustenstoß effektiver zu machen.

C Geräte zur Insufflation/Exsufflation können bei nicht-invasiv und bei invasiv beatmeten Patienten eingesetzt werden.

D Apparative Hustenhilfen dürfen erst nach der ersten Pneumonie verordnet werden.

E Zur Reduktion der Sekret- und Speichelbildung können Anticholinergika oder Botulinumtoxin A eingesetzt werden.

\section{Welche der folgenden Aussagen trifft nicht zu?}

A Die Messung der Vitalkapazität erfordert die Durchführung einer Bodyplethysmographie beim Lungenfacharzt.

B Die Messung der Vitalkapazität im Liegen liefert wichtige Hinweise auf eine mögliche Zwerchfellparese.

C Von paradoxer Atmung spricht man dann, wenn sich bei der Inspiration in Rückenlage die Thoraxexkursion von einer Absenkung der Bauchdecke begleitet wird.

D Die Messung des maximalen inspiratorischen und exspiratorischen Drucks kann mittels eines portablen Manometers erfolgen.

E Die Sonographie ist eine nicht-invasive Methode, um die Funktion des Zwerchfells zu untersuchen. 
Ohne nächtliches $\mathrm{CO}_{2}$-Monitoring kann die schlafbezogene Hypoventilation der poly(somno)graphischen Diagnostik entgehen..

B Die Polysomnographie mit Kapnometrie erlaubt die Zuordnung von Schlafstadien, $\mathrm{CO}_{2}$-Werten und peripherer Sauerstoffsättigung.

C Ein pathologischer Basenüberschuss in der morgendlichen Blutgasanalyse kann die renale Kompensation einer nächtlichen Hyperkapnie widerspiegeln.

D Die Durchführung stündlicher Blutgasanalysen während der Nacht ist eine gute Alternative zur kontinuierlichen transkutanen Kapnometrie.

E Im Gegensatz zur kardiorespiratorischen Polygraphie umfasst die kardiorespiratorische Polysomnographie die Ableitung des Schlaf-EEG.

8 Ein Patient mit primär bulbärer ALS berichtet über seit 2 Monaten regelmäßig auftretende Durchschlafstörungen und einen unerholsamen Nachtschlaf. Es werden die folgenden Messwerte erhoben: Vitalkapazität 54\% des Sollwertes, maximaler inspiratorischer Mundverschlussdruck $48 \mathrm{cmH}_{2} \mathrm{O}$, nächtliche Atemfrequenz $22 / \mathrm{min}$, mittlere Sauerstoffsättigung 95\%, Apnoe-Hypopnoe-Index 19,4/h, mittlerer transkutaner $\mathrm{CO}_{2}$-Wert $\left(\mathrm{tcCO}_{2}\right) \mathbf{4 8 , 6} \mathrm{mmHg}$, maximaler $\mathrm{tcCO}_{2} 53,0 \mathrm{mmHg}, \mathrm{pCO}_{2}$ am Tag $39,4 \mathrm{mmHg}$. Welche der folgenden Aussagen ist richtig?

A Eine nicht-invasive Heimbeatmung ist noch nicht indiziert, weil die mittlere Sauerstoffsättigung normal ist.

B Eine nicht-invasive Heimbeatmung ist noch nicht indiziert, weil der mittlere $\mathrm{tcCO}_{2}$ in der Nacht unter $50 \mathrm{mmHg}$ liegt.

C Eine nicht-invasive Heimbeatmung ist noch nicht indiziert, weil tagsüber keine Hyperkapnie besteht..

D Bei der Einleitung der nicht-invasiven Beatmung ist zu berücksichtigen, dass bei dem Patienten auch ein obstruktives Schlafapnoe-Syndrom vorliegt.

E Aufgrund der primär bulbären Verlaufsform der ALS ist die nicht-invasive Beatmung kontraindiziert.
A Symptome schlafbezogener Atmungsstörungen umfassen morgendliche Kopfschmerzen, Durchschlafstörungen und eine gesteigerte Tagesschläfrigkeit..

B Eine Zwerchfellschwäche kann sowohl neurogene als auch myogene Ursachen haben.

C Die myotone Dystrophie Typ 1 kann mit sämtlichen Subtypen schlafbezogener Atmungsstörungen einhergehen.

D Patienten mit NME und nächtlicher Hyperkapnie, die nicht über Schlafstörungen oder eine vermehrte Tagesschläfrigkeit berichten, bedürfen keiner nicht-invasiven Beatmungstherapie.

E Eine zentrale Schlafapnoe mit Cheyne-Stokes-Atmung kann bei Patienten mit schwerer dilatativer Kardiomyopathie und Herzinsuffizienz auftreten.

Welche der nachfolgenden Aussagen ist nicht zutreffend?

Ein Hustenspitzenstoß über 270 1/min ist als normal anzusehen.

B Bei der Berechnung des Sollwerts der Vitalkapazität werden Geschlecht, Alter und Körpergröße berücksichtigt.

C Zu den nicht mitarbeitsabhängigen Methoden zur Untersuchung der Zwerchfellfunktion gehören die Sonographie, die Phrenicusneurographie und die bilaterale Magnetstimulation des N. phrenicus.

D Die kardiorespiratorische Polysomnographie umfasst im Gegensatz zur Polygraphie u. a. die Ableitung des SchlafEEG.

E Die Körperposition hat keinen Einfluss auf die Ventilation. 